\title{
Anti-CEA BiTE Monoclonal Antibody AMG211
}

National Cancer Institute

\section{Source}

National Cancer Institute. Anti-CEA BiTE Monoclonal Antibody AMG211. NCI Thesaurus.

Code 195728

A recombinant, proprietary bispecific T-cell engagers (BiT E) antibody directed against human carcinoembryonic antigen (CEA), with potential immunostimulating and antineoplastic activities. Anti-CEA BiTE monoclonal antibody AMG211 possesses two antigen-recognition sites, one for CEA and one for the CD3 complex, a group of T cell surface glycoproteins that complex with the T cell receptor (TCR). This bispecific monoclonal antibody brings CEA-expressing tumor cells and cytotoxic T lymphocytes (CT Ls) and helper T lymphocytes (HTLs) together, which may result in the CT L- and HTLmediated cell death of CEA-expressing cells. CEA, a tumor associated antigen, is overexpressed in many cancer types, including gastrointestinal, breast, non-small cell lung, and thyroid cancers. 